Arteterapia. Papeles de arteterapia y educación para inclusión social ISSN: $1886-6190$

\title{
Intervención con Arteterapia en alumnado de Primaria con déficits de atención
}

\author{
Mª Carmen Delgado Morán ${ }^{1}$; $\mathrm{M}^{\mathrm{a}}$ Nieves Pérez Marfi12; Francisco Cruz Quintana ${ }^{3}$
}

Recibido: 20 de julio de 2016 / Aceptado: 5 de octubre de 2016

Resumen. Se presentan los resultados de un estudio sobre el efecto de un programa de Arteterapia en alumnos de educación primaria que presentan dificultades de atención con, y sin, hiperactividad. Han participado 52 alumnos/as de centros escolares de Granada (España), distribuidos en dos grupos: grupo de intervención, formado por 32 alumnos/as que siguieron un programa con 10 sesiones de arteterapia y, grupo control formado por 20 alumnos/as. Los resultados muestran diferencias significativas en el grupo que participó en el programa, en las áreas de hiperactividad, de comportamiento, en el trastorno combinado de atención e hiperactividad y en percepción de diferencias.

Palabras clave: Arteterapia; Déficit de atención; Hiperactividad; Educación Primaria; TDAH.

\section{[en] Art Therapy intervention on Primary School pupils suffering from Attention difficulties}

\begin{abstract}
We present here the results of an Art Therapy program carried out with Primary School children suffering from Attention difficulties, with or without hyperactivity. In this study 52 pupils from two different schools in Granada (Spain) took part. 32 out of them formed the experimental group and attended 10 Art Therapy sessions. The other 20 children formed the control group. Results have shown significant differences on children taking part in the Art Therapy program, and mainly in the areas of hyperactivity, behavior disorder, as well as in attention and hyperactivity disorder and perception of differences.
\end{abstract}

Keywords: Art Therapy; attention deficit- disorder; hyperactivity disorder; elementary education; ADHD.

1 Psicóloga, Psicoterapeuta, Arteterapeuta y docente en Arteterapia. Artículo referido a la investigación realizada en el marco del Departamento de Personalidad, Evaluación y Tratamiento Psicológico. Facultad de Psicología. Universidad de Granada.

E-mail: mcarmendelgado9@gmail.com

2 Docente del Departamento de Personalidad, Evaluación y Tratamiento Psicológico. Facultad de Psicología. Universidad de Granada

E-mail: nperez@ugr.

3 Docente del Departamento de Personalidad, Evaluación y Tratamiento Psicológico. Facultad de Psicología. Universidad de Granada

E-mail: fcruz@ugr.es 
Sumario. 1. Introducción; 2. Método: Participantes; 3. Instrumentos; 4. Programa de intervención; 5. Procedimiento; 6. Diseño y análisis de datos; 7. Cuestiones éticas; 8. Resultados; 9. Discusión; 10. Referencias bibliográficas.

Cómo citar: Delgado Morán, $\mathrm{M}^{\mathrm{a}}$ C.; Pérez Marfil, M ${ }^{\mathrm{a}}$ N.; Cruz Quintana, F. (2016) Intervención con Arteterapia en alumnado de Primaria con déficits de atención, en Arteterapia. Papeles de arteterapia y educación para inclusión social 11, 277-291.

\section{Introducción}

Existe una abundante evidencia empírica, basada en estudios de casos, que documenta la utilidad de las terapias de artes creativas (TAC) y entre ellas el Arteterapia, en diferentes ámbitos, aunque el número de estudios experimentales controlados, publicados sobre sus efectos y los mecanismos de acción, es todavía muy escaso si se compara con otras técnicas de intervención (Collette, 2011; Goodill, 2010; Pratt, 2004). Hay consenso en considerar que es a partir de mediados del siglo veinte, cuando se produce una concurrencia de circunstancias, que facilitan la aparición del Arteterapia como posibilidad terapéutica (Sánchez, 2006).

En la actualidad, se utiliza como una opción terapéutica dirigida a mejorar el bienestar psicológico de la persona mediante el uso de diferentes intervenciones en las que se utilizan los materiales y procedimientos plásticos, dándole mayor importancia al proceso de creación y a las cuestiones que surgen en dicho proceso, que a las imágenes finales producidas (López-Martínez, 2009). Las actividades creativas siempre han sido relevantes, entre otros contextos, en salud mental. Existe un creciente reconocimiento de que tienen un papel importante en la mejora de la salud y el bienestar de los individuos (Leckey, 2011), además de contribuir significativamente a la humanización y al confort de las modernas instituciones de salud, aliviando el estrés, la ansiedad y el dolor de los pacientes y cuidadores (Goodill, 2010).

Las aplicaciones del Arteterapia son diversas (Del Río, 2009), aunque su uso se está extendiendo, sobre todo, en los ámbitos: sanitario (Öster, 2006; Rico, 2007; Monti, 2006), educativo (Gallardo et al., 2010; Mateos, 2004) y social (Delgado, 2009; Ojeda y Serrano, 2008).

Las investigaciones al respecto han encontrado que la participación en la actividad creativa se asocia con una mejor salud mental, reduciendo tanto síntomas auto-informados, como manifestaciones clínicas (Caddy, Crawford y Page, 2012). Las aplicaciones en población clínica adulta ponen de manifiesto la utilidad de estas técnicas, por ejemplo, en pacientes diagnosticados de esquizofrenia (Patterson, Debate, Anju, Waller y Crawford, 2011), en madres con depresión leve y moderada postnatal (Perry, Thurston y Osborn, 2008), en pacientes con enfermedad de Alzheimer (Guetin et al., 2009), y en los efectos de los tratamientos y manejo de síntomas oncológicos (Monti et al., 2006; Öster et al., 2006; Svensk et al., 2009; Wood, Molassiotis y Payne, 2011), entre otros.

En población clínica infantil, la literatura informa de la obtención de buenos resultados en la aplicación de técnicas de Arteterapia en niños con tumores 
cerebrales y-tratamientos con quimioterapia (Madden, Mowry, Gao, Cullen y Foreman, 2010), control del dolor en niños con leucemia sometidos a procedimientos dolorosos, como punción lumbar y aspiración de médula ósea (Favara-Scacco, Smirne, Schilirò y Di Cataldo, 2001), trastornos psicomotores (Araujo y Gabelán, 2010) y diabetes (Basso y Pelech, 2008).

Por otra parte, los estudios que han analizado los beneficios de las técnicas de Arteterapia en el ámbito escolar, han encontrado que favorecen la integración multicultural (Omenat, 2003), estimula la imaginación y el pensamiento creativo aumenta la autoestima, fomenta las habilidades sociales (Rodríguez, 2007) y previene problemas emocionales y de comportamiento en niños inmigrantes y refugiados (Rousseau et al., 2005).

Sin embargo, pocos trabajos han tratado de comprobar el efecto de programas de arteterapia en niños con problemas de atención y, los que lo han hecho, presentan problemas metodológicos. La dificultad a la hora de prestar atención de los niños y niñas en edad escolar es un problema que preocupa actualmente tanto a investigadores como al profesorado y a las familias. A veces, de forma aislada y, otras veces, acompañado de hiperactividad, impulsividad o trastornos del comportamiento, es un problema clínico que afecta a la conducta y, de forma muy grave, al aprendizaje escolar. Además, suelen producirse comportamientos disruptivos y dificultades en el área verbal, en el razonamiento y en el cálculo. El $37 \%$ de los alumnos con dificultades de atención, presentan también TDAH (Trastorno por déficit de atención e hiperactividad) (González-Castro, Álvarez, Núñez, González-Pienda y Álvarez, 2010).

Los síntomas principales que se describen en este trastorno son: un nivel inapropiado en atención o hiperactividad, movimientos excesivos en situaciones inapropiadas, pérdida de atención de forma sostenida, alteraciones en la función ejecutiva y los procesos de inhibición y tiempos de respuesta inapropiados debido a las dificultades en la función de corrección del error (Martínez-León, 2006). Estos síntomas suelen aparece antes de los 12 años de edad y se detectan en diferentes ámbitos de la vida del niño (DSM 5, 2014)

En los estudios realizados, se observa una incidencia del trastorno de entre un $3 \%$ y un $5 \%$ de los niños, con mayor frecuencia en varones, y es la causa más importante de fracaso escolar y desajuste social (López-Villalobos et al., 2004).

Se han descrito, principalmente, dos líneas de investigación en este campo. La primera de ellas, liderada por Brown (2000), considera que existe un continuo a nivel neurológico, entre el TDA (Trastornos por Déficit de Atención) y el TDAH. La segunda, representada por Barkley (1998), se centra fundamentalmente en el estudio de las dificultades en la inhibición y de la función ejecutiva, y considera que el TDA y el TDAH son dos categorías independientes.

Un amplio número de trabajos documentan que el TDAH se caracteriza por déficit en la función ejecutiva, y que los niños que sufren este trastorno tienen un rendimiento más pobre en un rango de tareas relacionadas con las funciones ejecutivas, en comparación con participantes controles. Un meta-análisis de 83 estudios en los cuales se mide función ejecutiva en niños y adolescentes con TDAH, ha encontrado que los participantes con TDAH, muestran deterioro significativo en algunas medidas neuropsicológicas como son la inhibición de respuesta, vigilancia, memoria de trabajo y planificación (Wilcutt et al., 2005). 
En el espacio educativo movido por la innovación y por la comunicación verbal, oral o escrita, como formas privilegiadas de expresión, hay cada vez más alumnos que no siguen el ritmo escolar por el hecho de no poder prestar atención y, cada vez, también, son más las opiniones que manifiestan la necesidad de establecer programas que favorezcan otros sistemas de trabajo con alumnos con necesidades educativas especiales (Zubillaga y Pastor, 2005). Por todo lo anteriormente expuesto, el objetivo de este trabajo es comprobar el efecto de un programa de intervención con Arteterapia en un grupo de alumnos que cursan educación primaria y presentan dificultades de atención. La hipótesis de la que se parte es que el programa de Arteterapia mejorará las dificultades atencionales, el autoconcepto, la creatividad, el bienestar emocional y los problemas de comportamiento en este grupo de alumnos.

\section{Método}

\section{Participantes}

Los participantes, han sido derivados al programa por los profesores-tutores, el Equipo de Apoyo a la Integración y el Servicio de Orientación de los dos centros educativos en los que se realizó el estudio. El alumnado elegido para formar parte de la muestra, se seleccionó de acuerdo a la puntuación obtenida en el Cuestionario EDAH (Evaluación del trastorno por déficit de atención con hiperactividad), en la escala de dificultades atencionales, siendo seleccionados los que presentaban puntuaciones más elevadas. El grupo de tratamiento $(n=32)$ lo formaron, además, aquellos niños/as con mayor deterioro/riesgo y, el resto de alumnos seleccionados, formaron el grupo control (en condición de lista de espera) $(n=20)$.

Las edades de los alumnos se encontraban comprendidas entre los 7 y los 12 años $(\mathrm{ME}=9.57, \mathrm{DT}=1)$. En el grupo de tratamiento participaron 15 niñas $(46.88 \%)$ y 17 niños $(53.12 \%)$, y en el grupo control, 7 niñas $(35 \%)$ y 13 niños $(65 \%)$.

\section{Instrumentos}

a) Evaluación del trastorno por déficit de atención con hiperactividad, EDAH (Farré y Narbona, 2003)

Consta de 20 ítems con respuestas que van del 0 al 3, según la frecuencia con la que el profesor observara la conducta. Las puntuaciones determinan la existencia de dos niveles de riesgo de padecer el trastorno (riesgo moderado y riesgo elevado) y otro nivel sin riesgo. La fiabilidad de la prueba se ha establecido mediante el alpha de Cronbach y todas las subescalas presentan coeficientes iguales o superiores a .90. En el caso de la validez, las correlaciones entre las puntuaciones de la prueba y los criterios del DSM-IV para el diagnóstico del Déficit de Atención ofrecen una puntuación de .745.

b) Cuestionario de Autoevaluación Ansiedad Estado/Rasgo para niños, STAIC (Spielberger et al., 1970) (Adaptación Seisdedos, 1990)

Este instrumento analiza por separado la "ansiedad-estado" (A-E), que evalúa estados transitorios de ansiedad y la "ansiedad-rasgo" (A-R), que informa sobre 
diferencias relativamente estables de propensión a la ansiedad. La fiabilidad y validez de este instrumento han sido demostradas en trabajos previos (Pons-Salvador, Frías y Del Barrio, 1994).

c) Cuestionario de Autoconcepto Garley, CAG (García, 2001).

La prueba consta de 48 afirmaciones relacionadas con 6 dimensiones del autoconcepto: físico, social, familiar, intelectual, personal y sensación de control. Esta última dimensión resulta novedosa en las pruebas de autoconcepto para estas edades. La fiabilidad de la prueba mediante el coeficiente de Cronbach es de .87. La validez convergente y de criterio, se ha realizado mediante correlaciones en tres ámbitos: con una prueba sociométrica diseñada ad oc y con ciertas capacidades intelectuales.

d) Cuestionario "Big Five” de personalidad para niños y adolescentes, BFQ-NA (Barbaranelli, Caprara y Rabasca, 2006)

La adaptación española ha sido realizada en 2006 (Del Barrio, Carrasco y Holgado, 2006). La finalidad de esta prueba es evaluar la personalidad de niños y adolescentes de acuerdo con la teoría de los Cinco Grandes factores de la personalidad: Inestabilidad emocional (Ie), Extraversión (Ex), Apertura (Ap), Amabilidad (Am) y Conciencia (Co). Incluye un total de 65 elementos, con formato autoinformado, que se valoran mediante una escala de cinco alternativas. Los estudios de fiabilidad mediante el alpha de Cronbach muestran unas puntuaciones que oscilan entre .70 para la Inestabilidad emocional y .88 para el factor Conciencia.

e) Test de percepción de diferencias, CARAS (Thurstone y Yela, 2001).

Evalúa la aptitud para percibir, rápida y correctamente, semejanzas y diferencias y patrones estimulantes ordenados parcialmente. Consta de 60 elementos gráficos, cada uno compuesto por tres posibilidades de respuesta de la que sólo una es la correcta. Los estudios de fiabilidad ofrecen diferentes puntuaciones según el método utilizado: .60 con el procedimiento test-retest y .97 con el de las dos mitades. La validez medida con una batería de criterios externos de éxito en el trabajo dio una correlación de 69 .

f) Inteligencia creativa, CREA (Corbalán, et al., 2003).

La finalidad de esta prueba es la apreciación de la inteligencia creativa a través de una evaluación cognitiva de la creatividad individual, según el indicador de generación de cuestiones, en el contexto teórico de búsqueda y solución de problemas. Se presenta con tres formas: A, B y C. La fiabilidad del test se ha medido mediante el coeficiente de equivalencia entre las formas $\mathrm{A}$ y $\mathrm{B}$ presentando un valor de .875. La validez concurrente obtenida través de la correlación múltiple entre la Batería de Creatividad de Guilford (fluidez, flexibilidad, originalidad y producción divergente) y las distintas formas del CREA, ofrecen resultados de .79 para el CREA-A y de .81 para el CREA-B.

\section{Programa de intervención}

Con los alumnos seleccionados para el grupo de intervención se formaron cuatro grupos, dos en cada centro escolar, teniendo en cuenta el curso al que pertenecían. Así los niños que estaban entre $2^{\circ}$ y $4^{\circ}$ de Primaria formaron un grupo y, los niños de $5^{\circ}$ y $6^{\circ}$ de Primaria formaban el otro grupo.

Se llevaron a cabo 10 sesiones con cada uno de los subgrupos de intervención, 40 sesiones en total. La duración de cada sesión fue de 55 minutos. Se ofreció el mismo tipo de material artístico y el mismo tema de trabajo para cada grupo en 
cada sesión, siendo cada alumno el que elegía el material con el que deseaba representar su obra.

Los materiales que se proporcionó a los alumnos fueron: lápices y rotuladores de colores, diferentes clases de pinturas, pinceles, tijeras, cartulinas, diferentes clases de papel, cola, plastilina, revistas y cartón.

En la primera sesión del programa, se informó de las normas de trabajo del grupo: respeto por el otro y por su obra, respeto con los materiales, libertad de expresión y confidencialidad.

El esquema seguido en las sesiones de intervención fue el siguiente:

1. Elección del sitio en el aula por parte de los alumnos.

2. Indicación de la "Propuesta de trabajo" de la sesión.

3. Elección del material para realizar la obra.

4. Elaboración de la obra de forma individual o grupal según se indicara.

5. Presentación al grupo, del trabajo artístico personal con la designación de un título y exposición oral (voluntaria) del trabajo realizado.

Las propuestas de trabajo se han concretado para cada una de las 10 sesiones: "Presentación"," Escoge un lugar", "Propuesta libre”, "Trabajo en grupo", "Realiza un disfraz o careta", "Representa un sueño", "Representa una ilusión o deseo", "Un regalo", "Mi colegio", "Despedida".

La temática particular de las sesiones se engloban dentro de las siguientes grandes áreas: Autoconcepto, Relaciones Interpersonales, Autobiografía, Inquietudes y Deseos (López, 2009).

\section{Procedimiento}

En una primera fase, se contactó con los centros participantes en el estudio y se informó a los directores de la actuación que se iba a realizar. Una vez conseguido el permiso, se solicitó del profesorado de Primaria de cada Centro, del Equipo de Apoyo a la Integración y del Servicio de Orientación, la selección de los posibles participantes, de acuerdo a sus dificultades de atención en clase. Se reunió al grupo de alumnos que iban a participar en el estudio y, por medio de los profesores, se solicitó el consentimiento informado de los padres o tutores de los niños.

Posteriormente se inició el programa de acuerdo a las siguientes fases:

Fase 1. Selección. De acuerdo a lo señalado en la descripción de los participantes.

Fase 2. Evaluación. Se realiza una primera evaluación inicial de todos los grupos implicados en la investigación: intervención y controles. Dicha evaluación se realiza con los instrumentos descritos anteriormente, y en el siguiente orden: STAIC, CAG, BFQ-NA, CARAS y CREA.

Fase 3. Intervención. Los alumnos se citaban a una hora prefijada destinada para las sesiones, tras consensuarlo con el profesorado implicado. Las sesiones de terapia se han desarrollado en los espacios facilitados por los centros participantes. Han transcurrido a lo largo del tercer trimestre, dos sesiones de Arteterapia por 
semana, para cada grupo, durante 5 semanas y todas las sesiones han sido realizadas por la misma psicóloga experta en arteterapia.

Fase 4. Evaluación post intervención. Se realizó una nueva evaluación con los mismos instrumentos descritos, excepto el BFQ-NA y el STAIC-R que evalúan rasgos de personalidad.

\section{Diseño y análisis de los datos}

Se ha utilizado un diseño cuasi-experimental de medidas repetidas prepostratamiento con grupo control en condición de lista de espera. El tratamiento de los datos se ha realizado utilizando el paquete estadístico SPSS versión 15.0.

Para el análisis descriptivo de las variables sexo, edad, curso y de las diferentes escalas utilizadas se calcularon medias, desviaciones típicas y porcentajes. Se compararon las diferencias entre los grupos en las escalas citadas y entre el pre- y postratamiento utilizando la prueba $t$ de Student, para muestras independientes y muestras relacionadas, respectivamente. El intervalo de confianza usado fue del $95 \%$.

\section{Cuestiones éticas}

Dado que el colectivo en estudio se refiere a niños pequeños y, en todo caso, menores de edad, se han solicitados los permisos por parte de la familia y se ha protegido la imagen para evitar la posible identificación, en cualquier documento visual realizado.

\section{Resultados}

En primer lugar se presentan los resultados de los análisis de las diferencias entre los grupos antes y después de la intervención (Ver tabla 1). Previa a la intervención, los resultados muestran que existen diferencias significativas en todas las escalas del EDAH (Hiperactividad: $t=2.019, p=.049$; Atención: $t=5.272$, $p=.000$; Trastornos de conducta: $t=2.707, p=.009$ e Hiperactividad-Atención: $t=$ $3.974, p=.000)$, en el número de aciertos en CARAS $(t=-5.06, p=.000)$, Ansiedad Estado del STAI-C $(t=2.059, p=.045)$, CAG Social $(t=-2.259, p=.028)$ y el factor de Inestabilidad Emocional del BFQ-NA $(t=-2.297, p=.027)$. Estos datos indican valores más altos para el grupo de intervención en las escalas del EDAH, la Ansiedad Estado, y valores más bajos que los del grupo control en aciertos en CARAS, CAG Social e Inestabilidad Emocional.

Los resultados obtenidos después de la intervención realizada para todas las variables anteriores, excepto para las variables de rasgo (STAI-R y factores del BFQ-NA),-muestran diferencias significativas entre los grupos en las escalas de Atención $(t=5.152, p=.000)$, Trastornos de Conducta $(t=2.102, p=.041) \mathrm{e}$ Hiperactividad-Atención $(t=4.176, p=.000)$ del EDAH. Son los niños del grupo de tratamiento los que presentan las puntuaciones más altas en estas escalas. No se encuentran diferencias en el resto de las medidas. 
Tabla 1. Prueba $t$ para las diferencias entre el grupo tratamiento y control en los valores pre y postratamiento $(N$ Tratamiento $=32, N$ Control $=20)$

\begin{tabular}{lll}
\hline & Pre-tratamiento & Postratamiento \\
\hline EDAH & & \\
Hiperactividad & $2.019^{* *}$ & 1.436 \\
Atención & $5.272^{* * *}$ & $5.152^{* * *}$ \\
$\begin{array}{l}\text { Trastornos de } \\
\text { Conducta }\end{array}$ & $2.707^{* * *}$ & $2.102^{* *}$ \\
Hiperacividad- & $3.974^{* * *}$ & $4.176^{* * *}$ \\
Atención & & \\
CARAS & & \\
& $-5.06^{* * *}$ & -.158 \\
CREA & & \\
& -1.534 & .857 \\
STAIC & & \\
Estado & & \\
Rasgo & $2.059^{* *}$ & 1.818 \\
CAG & .362 & \\
Físico & & \\
Social & -1.305 & -.948 \\
Familiar & $-2.259^{* *}$ & -1.923 \\
Intelectual & -1.855 & -.680 \\
Personal & -1.259 & -1.220 \\
& -1.101 & -1.103 \\
BFQ-NA & & \\
Conciencia & 1.355 & \\
Apertura & .377 & \\
Extraversión & .978 & \\
Amabilidad & -.516 & \\
Inestabilidad & $-2.297^{* *}$ & \\
Emocional & & \\
\hline
\end{tabular}

Nota. EDAH $=$ Evaluación del trastorno por déficit de atención con hiperactividad; CREA $=$ Inteligencia creativa; CARAS $=$ Test de percepción de diferencias; STAIC = Cuestionario de autoevaluación ansiedad Estado/Rasgo para niños $; \mathrm{CAG}=$ Cuestionario de autoconcepto Garley ; BFQ-NA $=$ Cuestionario "Big Five" de personalidad para niños y adolescentes.

$* * p<.05 ; * * * p<.01$

En la Tabla 2 se presentan los resultados de los análisis de las diferencias pre-post intervención de acuerdo con la $t$ de Student. Los datos obtenidos reflejan que no aparece ninguna diferencia en las comparaciones pre y post para el grupo control. Por el contrario, sí se encuentran diferencias significativas en el grupo de tratamiento en las escalas de Hiperactividad $(t=3.131, p=.004)$, Trastornos de Conducta $(t=$ 5.506, $p=.000)$ e Hiperactividad/Atención $(t=2.707, p=.011)$ del EDAH, así como en el número de aciertos de CARAS $(t=-6.613, p=.000)$. Las medias señalan una 
disminución tras el tratamiento en los valores de las escalas del EDAH, y un aumento en el número de aciertos de la prueba de CARAS.

Tabla 2. Medias y DT (entre paréntesis) del grupo de tratamiento y del grupo control en el pre y el postratamiento, valor de $t$ y tamaño del efecto ( $N$ Tratamiento $=32, N$ Control $=20$ )

\begin{tabular}{|c|c|c|c|c|c|}
\hline & & $\begin{array}{l}\text { Pre- } \\
\text { tratamiento }\end{array}$ & Postratamiento & $t$ & $\begin{array}{l}\text { Tamaño } \\
\text { del } \\
\text { efecto }\end{array}$ \\
\hline \multicolumn{6}{|l|}{ EDAH } \\
\hline \multirow[t]{2}{*}{ Hiperactividad } & G. Trata & $7.06(4.54)$ & $6.09(3.90)$ & $3.131 * * *$ & .230 \\
\hline & G. Contr & $4.50(4.31)$ & $4.55(3.56)$ & -.105 & .013 \\
\hline \multirow[t]{2}{*}{ Atención } & G. Trata & $11.25(3.05)$ & $10.75(2.91)$ & 1.559 & .168 \\
\hline & G. Contr & 6.0 & $5.85(3.94)$ & .977 & .211 \\
\hline \multirow{2}{*}{$\begin{array}{l}\text { Trastornos de } \\
\text { conducta }\end{array}$} & G. Trata & $10.75(7.89)$ & $9.18(7.84)$ & $5.506 * * *$ & .200 \\
\hline & G. Contr & $4.90(7.04)$ & $4.85(6.13)$ & .098 & .008 \\
\hline Hiperacividad- & G. Trata & $18.31(6.74)$ & $16.84(5.45)$ & $2.707 * *$ & .241 \\
\hline Atención & G Contr & $11.10(5.70)$ & $10.40(5.35)$ & .679 & .127 \\
\hline \multirow[t]{2}{*}{ CARAS } & G. Trata & $20.13(9.42)$ & $33.75(10.10)$ & $-6.613 * * *$ & -1.395 \\
\hline & G. Contr & $33.55(9.10)$ & $34.16(6.39)$ & -.419 & -.079 \\
\hline \multirow[t]{2}{*}{ CREA } & G. Trata & $9.22(5.22)$ & $10.63(6.98)$ & -1.776 & -.231 \\
\hline & G. Contr & $11.32(3.61)$ & 12.16 & -.837 & -.208 \\
\hline \multicolumn{6}{|l|}{ STAIC } \\
\hline \multirow[t]{2}{*}{ Estado } & G. Trata & $33.75(6.79)$ & $33.69(6.87)$ & .047 & .009 \\
\hline & G. Contr & $29.80(6.64)$ & $30.05(7.25)$ & -.124 & -.036 \\
\hline \multicolumn{6}{|l|}{ CAG } \\
\hline \multirow[t]{2}{*}{ Físico } & G. Trata & $31.13(6.91)$ & $31.59(6.33)$ & -.541 & -.069 \\
\hline & G. Contr & $33.60(6.21)$ & $33.26(5.64)$ & .276 & .057 \\
\hline \multirow[t]{2}{*}{ Social } & G. Trata & $26.22(4.95)$ & $26.44(4.54)$ & -.261 & -.046 \\
\hline & G. Contr & $29.15(3.82)$ & $28.90(4.18)$ & .382 & .062 \\
\hline \multirow[t]{2}{*}{ Familiar } & G. Trata & $23.13(3.45)$ & $23.75(4.38)$ & -.793 & -.158 \\
\hline & G. Contr & $24.78(4.18)$ & $24.56(3.28)$ & .356 & .059 \\
\hline \multirow[t]{2}{*}{ Intelectual } & G. Trata & $27.72(4.70)$ & $28.19(5.20)$ & -.494 & -.095 \\
\hline & G. Contr & $29.25(3.45)$ & $29.90(4.12)$ & -.609 & -.177 \\
\hline \multirow[t]{2}{*}{ Personal } & G. Trata & $27.75(4.96)$ & 27.78 (3.69) & -.039 & .007 \\
\hline & G. Contr & $29.20(3.99)$ & $28.89(3.11)$ & .567 & .087 \\
\hline
\end{tabular}

Nota. EDAH $=$ Evaluación del trastorno por déficit de atención con hiperactividad; CREA = Inteligencia creativa $;$ CARAS $=$ Test de percepción de diferencias; STAIC = Cuestionario de autoevaluación ansiedad Estado/Rasgo para niños $; \mathrm{CAG}=$ Cuestionario de autoconcepto Garley; G.Trat $=$ Grupo Tratamiento; G. Contr $=$ Grupo Control . ** $p<.05 ; * * * p<.01$

Por último, en la Tabla 3 se presenta la clasificación de los niños del grupo de tratamiento en los distintos grupos de riesgo que establece la prueba EDAH (sin riesgo, riesgo moderado y riesgo alto), para cada una de las escalas. Para hacer la clasificación se han tenido en cuenta los cursos a los que pertenece cada alumno, 
utilizando los baremos apropiados en cada caso. Al comparar los resultados del pre-tratamiento con los posteriores a la intervención, se aprecia un cambio en la distribución de los participantes. En la segunda evaluación podemos observar que el grupo de riesgo alto ha bajado en todas las escalas, es decir, hay menos niños con dificultades extremas. De la misma manera, aumentan los niños que se pueden encuadrar en el grupo sin riesgo.

Tabla 3. Clasificación del grupo de intervención en función del riesgo en las diferentes escalas del EDAH

\begin{tabular}{lcccccc}
\hline & \multicolumn{3}{c}{ PRETRATAMIENTO } & \multicolumn{3}{c}{ POSTRATAMIENTO } \\
& $\begin{array}{l}\text { Sin } \\
\text { riesgo }\end{array}$ & $\begin{array}{l}\text { Riesgo } \\
\text { moderado }\end{array}$ & $\begin{array}{l}\text { Riesgo } \\
\text { alto }\end{array}$ & $\begin{array}{l}\text { Sin } \\
\text { riesgo }\end{array}$ & $\begin{array}{l}\text { Riesgo } \\
\text { moderado }\end{array}$ & $\begin{array}{l}\text { Riesgo } \\
\text { alto }\end{array}$ \\
\hline Hiperactividad & 22 & 1 & 9 & 23 & 4 & 5 \\
& $(68.8 \%)$ & $(3.1 \%)$ & $(28.1 \%)$ & $(71.9 \%)$ & $(12.5 \%)$ & $(15.6 \%)$ \\
Atención & 4 & 10 & 18 & 8 & 11 & 13 \\
& $(12.5 \%)$ & $(31.3 \%)$ & $(56.3 \%)$ & $(25 \%)$ & $(34.4 \%)$ & $(40.6 \%)$ \\
Trastornos de & 19 & 7 & 6 & 24 & 3 & 5 \\
conducta & $(59.4 \%)$ & $(21.9 \%)$ & $(18.8 \%)$ & $(75 \%)$ & $(9.4 \%)$ & $(15.6 \%)$ \\
Hiperactividad- & 13 & 7 & 12 & 15 & 9 & 8 \\
atención & $(40.6 \%)$ & $(21.9 \%)$ & $(37.5 \%)$ & $(46.9 \%)$ & $(28 \%)$ & $(25 \%)$ \\
\hline
\end{tabular}

\section{Discusión}

El objetivo de este trabajo de investigación ha sido comprobar los efectos de un programa de intervención con Arteterapia en un grupo de alumnos que cursan educación primaria y presentan dificultades de atención.

Los resultados señalan que el programa de intervención desarrollado ha influido de forma positiva en la mejora de variables que son básicas para el aprendizaje y se muestra beneficioso para su aplicación en contextos escolares. Estos datos están en consonancia con investigaciones previas que han mostrado cómo los programas de Arteterapia son útiles para intervenir de manera terapéutica con determinadas poblaciones clínicas y con poblaciones que presentan problemas sociales (Alcaide, 2006; Araujo y Gabelán, 2010)

Si se analizan los efectos de la intervención sobre cada una de las variables estudiadas, se han encontrado diferencias estadísticamente significativas entre el grupo de intervención y el grupo control, en variables que afectan al rendimiento escolar, como son: la atención, la hiperactividad o las dificultades en el comportamiento. El programa de Arteterapia, ha producido una mejora de la atención y una disminución de la Hiperactividad, encontrándose diferencias significativas en el grupo de tratamiento en las escalas de Hiperactividad, Trastornos de Conducta, e Hiperactividad/Atención del EDAH, así como en el número de aciertos de CARAS, el aumento en la percepción de diferencias. Estos resultados están en sintonía con los encontrados en el estudio de Gallardo et al. 
(2010) con un grupo de estudiantes mexicanos diagnosticados de TDAH, donde se informa de cambios cognitivos y conductuales a corto plazo, después de aplicar un programa de Arteterapia. Sin embargo, los propios autores reconocen las limitaciones de su trabajo, debido al tamaño de la muestra (13 escolares) y por el hecho de no incluir un grupo control. Estas dificultades, se han intentado solventar en el presente trabajo, aumentando la muestra de alumnos e introduciendo un grupo control.

Respecto a los efectos del programa sobre el autoconcepto, los resultados no resultan significativos. En el estudio de Rousseau et al., (2005) con niños emigrantes y refugiados se informó de un efecto positivo sobre la autoestima y los problemas de comportamiento, con un programa realizado durante 12 semanas que incluyó un trabajo de modificación de las percepciones de los profesores hacia los recién llegados, colocando el énfasis en su fuerza y su capacidad de recuperación.

En este trabajo, sería de esperar que un alumno que mejora su estado atencional, que empieza a controlar su hiperactividad psicomotora y sus problemas de comportamiento, fuese, igualmente, mejorando la imagen de sí mismo. Hay que tener en cuenta que, por lo general, los alumnos que presentan estos problemas a nivel escolar, muestran dificultades, también a nivel social e incluso familiar, como refleja la literatura consultada (López-Villalobos, et al., 2004), cuestión que hace más compleja la percepción de sí mismo. Por otra parte, hay estudios que muestran que los cambios en el autoconcepto no aparecen de forma inmediata, sino que podrían ponerse de manifiesto más a largo plazo, ya que resulta necesario una toma de conciencia de los cambios producidos, no sólo por los propios participantes, sino también de la comunidad educativa para propiciar un mayor refuerzo positivo (Allen, 2003). En este trabajo no fue posible realizar una intervención posterior con el profesorado.

En referencia al efecto del programa de intervención sobre la mejora en la creatividad, también era de esperar un cambio significativo en el alumnado, al ser precisamente el modelo de terapia utilizado, una terapia de contenido artístico. En este caso, las puntuaciones sí se acercan a la significación y se observa una tendencia importante que no se refleja en el grupo control. Por tanto, puede decirse que, posiblemente, después de un tratamiento con Arteterapia con un mayor número de sesiones, podrían obtenerse resultados significativos en esta variable, ya que la tendencia estadística así lo indica.

Los datos de este trabajo son concordantes con los obtenidos por Duarte (2003) en un estudio realizado con niños con capacidades extraordinarias o sobredotación, niños con problemas de aprendizaje y niños con déficit de atención con hiperactividad. Los resultados mostraron que para los niños con necesidades educativas especiales, la creatividad representa un recurso psicológico importante para enfrentar los requerimientos educativos dentro del ámbito formal.

Por otra parte, los datos sugieren una ligera mejoría en la media del grupo sobre el que se ha intervenido y un ligero empeoramiento en la del grupo control. Estos datos son concordantes también con los obtenidos en el estudio de Rousseau et al. (2005), donde se informaba que la participación en el programa de intervención contribuía a reconstruir de manera significativa el mundo personal y, al mismo tiempo, fortalecer el vínculo del niño con el grupo. 
Existe evidencia suficiente sobre el efecto reductor de la ansiedad con la aplicación de Terapias creativas tanto en población clínica adulta, como infantil (Favara-Scacco et al., 2001; Guetin et al., 2008; Madden, et al., 2010; Walsh et al., 2007), sin embargo, en este estudio, no se han encontrado diferencias significativas sobre la ansiedad estado. Quizá este resultado puede deberse a las circunstancias en las que se desarrolló la última fase del estudio, ya que coincidió con el final de curso.

En cuanto al efecto del tratamiento en los problemas de comportamiento, los resultados muestran que el programa de Arteterapia ha favorecido una disminución de dichos problemas. Las diferencias entre los grupos, previas a la intervención, se reducen en la segunda evaluación. Las puntuaciones obtenidas indican que, a pesar que el grupo de intervención partía de una situación más desfavorable con una mayor proporción de alumnos en los grupos de riesgo alto y moderado, tras el tratamiento de Arteterapia, disminuye la frecuencia de niños dentro de estas dos categorías. Pensamos que estos resultados son interesantes, ya que se trata de una modificación en una serie de dificultades, que tiene una importante repercusión a nivel escolar y en el clima del aula. Estos datos están en consonancia con los mostrados, también, por el trabajo de Rousseau et al. (2005).

En lo que se refiere al grupo control, se observa que no ha existido mejoría en ninguna de las variables establecidas en este estudio, por lo que las diferencias obtenidas en el grupo de intervención, pueden atribuirse a la aplicación del programa de Arteterapia. Estos datos dan fuerza a las opiniones que manifiestan la necesidad de establecer programas que favorezcan otros sistemas de trabajo para alumnos que aprenden de forma diferente, tanto por dificultades personales, que impiden realizar un proceso de aprendizaje normalizado en el currículum escolar, como, en el otro extremo, por tener altas capacidades y no ser detectadas a tiempo. Con la población infantil, el uso de las terapias artísticas está particularmente justificado por la gran espontaneidad gráfica generalmente observada en los niños, así como por su menor capacidad evolutiva de expresar verbalmente sus emociones y sentimientos (Collette, 2011).

Se señalan, a continuación, algunas limitaciones de este estudio. Por un lado, no sabemos si la significación de los datos hubiera mejorado con un mayor número de sesiones, pero es algo que es conveniente comprobar en posteriores estudios. Por otro lado, el tamaño de la muestra, a pesar del esfuerzo que se ha realizado, es un aspecto mejorable, para favorecer la generalización de los resultados obtenidos. Sería también deseable medir el efecto del programa en el rendimiento académico. En este trabajo no se ha podido realizar, dado el período de implantación del programa y la dificultad que ha existido para que los profesores, en tan corto período de tiempo, hayan podido evaluar los efectos positivos del programa en el rendimiento académico de los alumnos. Por eso, sería deseable un periodo de implantación del programa superior a seis meses.

Para terminar, el Arteterapia se ha mostrado como una opción terapéutica valiosa y eficaz para el tratamiento de ciertas dificultades relacionadas con los procesos atencionales y de comportamiento, de tanta importancia para el aprendizaje dentro del contexto escolar. Por lo que no debería descartarse su implantación como programa específico de tratamiento, posiblemente dentro del aula de apoyo a la integración, para los alumnos que presenten estas dificultades. 


\section{Referencias bibliográficas}

Alcaide, C. (2006). La expresión artística como intervención educativo-terapéutica. En M. López-Cao (Coord.), Creación y posibilidad: Aplicaciones en el arte en la integración social (pp. 89-106). Madrid: Alianza.

Allen, P. B. (2003). Arteterapia. Guía del autodescubrimiento a través del arte y la creatividad. Madrid: Gaia.

American Psychiatric Association (2002). DSM-IV-TR Manual diagnóstico y estadístico de los trastornos mentales. Barcelona: Masson, S.A.

Araujo,G. Gabelán, G., Psicomotricidad y Arteterapia Revista Electrónica Interuniversitaria de Formación del Profesorado [en linea] 2010, 13 (Sin mes): [Fecha de consulta: 20 de julio de 2016] Disponible en: $<$ http://www.redalyc.org/articulo.oa?id=217015570026> ISSN

American Psychiatric Association (2014). DSM-5. Manual diagnóstico y estadístico de los trastornos mentales. Editorial Médica Panamericana. ISBN 9788498358100.

Barbaranelli,C., Caprara, G.V.y Rabasca, A. (2006). BFQ-NA: Cuestionario Big Five para niños y adolescentes (8-15 años). Madrid: TEA Ediciones.

Barkley R.A. (1998). Attention deficit hyperactivity disorder: A handbook for diagnosis and treatment. Nueva York: Guilford Press.

Basso, R.V. y Pelech, W.J. (2008). A creative arts intervention for children with diabetes. Part 2: evaluation. Journal Psychosocial Nursing Mental Health Service, 46, 25-28.

Brown, E. T. (2000). Attention-deficit disorders and comorbidities in children, adolescents, and adults. Washington, DC: American Psychiatric Press.

Caddy,L., Crawford, F.y Page, A.C. (2012). Painting a path to wellness: correlations between participating in a creative activity groups and improved measured mental health outcome. Journal Psychiatric Mental Health Nursing,19(4):285-377.

DOI: $10.1111 / j .1365-2850.2011 .01785$.

Collette, N. (2011). Arteterapia y cáncer. Psicooncología, 8, 81-99.

Corbalán, J. et al. ( 2003). CREA: Inteligencia creativa. Madrid: TEA Ediciones.

Del Barrio, Carrasco \& Holgado, (2006). BFQ-NA cuestionario de los Cinco Grandes para niños y adolescentes (adaptación a la población española). Madrid: TEA Ediciones.

Del Río, M. (2009). "Reflexiones sobre la praxis en Arte terapia" en Arteterapia. Papeles de Arteterapia y Educacion para la Inclusion Social Vol. 4 Páginas 17- 26.

Delgado, M.C. (2009). Maltrato infantil, prevención y detección en el ámbito educativo, una experiencia con técnicas de Arteterapia. Encuentros de Psicología, 22, 5-9.

Duarte, E. (2003). Creatividad como un recurso psicológico para niños con necesidades educativas especiales. Sapiens: Revista Universitaria de Investigación, 2, 13-32

Farré, A. y Narbona, J. (2003). EDAH. Evaluación del Trastorno por Déficit de Atención con Hiperactividad. Madrid: TEA Ediciones.

Favara-Scacco, C., Smirne, G., Schilirò. G., y Di Cataldo, A. (2001). Art therapy as support for children with leukemia during painful procedures. Medical Pediatric Oncology Journal, 36, 474-80.

Gallardo, G., Padrón, A.L., Martínez, M.C., Barragán, H.R., Passaye, A., García, R., y Aguilar, E. (2010). El arteterapia como tratamiento del trastorno por déficit de atención en una muestra de escolares mexicanos. Archivos de Neurociencias, 15, 77-83.

García. B. (2001). CAG: Cuestionario de Autoconcepto Garley, versión 1.0.Madrid: Instituto de orientación Psicológica, EOS. 
Guetin, S., et al. (2009). Effect of music therapy on anxiety and depression in patients with Alzheimer's type dementia: randomised, controlled study. Dementia and Geriatric Cognitive Disorders 28(1):36-46.

González-Castro, P., Álvarez, L., Núñez, J.C., González- Pienda, J.A. y Muñiz, J. (2010). Cortical activation and attentional control in ADAH subtypes. International Journal of Clinical and Health Psychology, 10, 23-39.

Goodill, S.W. (2010). The creative arts therapies: making health care whole. Minnesota Medicine, 93, 49-59.

Leckey, J. (2011). The therapeutic effectiveness of creative activities on mental well-being: a systematic review of the literature. Journal of Psychiatric and Mental Health Nursing, 18, 501-509. DOI: 10.1111/j.1365-2850.2011.01693.x.

López, M.A., (2012). Sentirse en casa. ARIADNE: un proyecto para la inclusión de personas migrantes a través del arte. Arteterapia: papeles de arteterapia y educación artística para la inclusión social,7, 119-139.

López-Martínez, M.D. (2009). La Intervención Arteterapéutica y su Metodología en el Contexto Profesional Español. Tesis doctoral, Universidad de Murcia, Murcia, España.

López-Villalobos, J. A., Serrano, I., Delgado, J., Ruiz, F., García, M. J., y Sánchez, M. I. (2004). Trastorno por déficit de atención con hiperactividad: una alteración psicopatológica con impacto multidimensional. Anales de Psiquiatría, 20, 205-210.

Madden J. R., Mowry, P., Gao, D., Cullen, P.M., y Foreman, N.K. (2010). Creative arts therapy improves quality of life for pediatric brain tumor patients receiving outpatient chemotherapy. Journal of Pediatric Oncology Nursing, 27, 133-145.

Martínez-León, N.C. (2006). Psicopatología del trastorno por déficit atencional e hiperactividad. International Journal of Clinical and Health Psychology, 6, 379-399.

Mateos, L.A. (2004). Actividades musicales para atender a la diversidad. Madrid: ICCE.

Monti, D.A., Peterson, C., Kunkel, E.J., Hauck, W.W., Pequignot, E., Rhodes, L., y Brainard, G.C. (2006). A randomized, controlled trial of mindfullnessbased art therapy (MBAT) for women with cancer. Psycho-Oncology, 15, 363- 373.

Ojeda, M. y Serrano, A.(2008). Mujer y Arteterapia. Una visión sobre la violencia de género. Arteterapia: papeles de arteterapia y educación artística para la inclusión social,3, 157-164.

Omenat, M. (2003). El sentido del Arteterapia en la escuela: una experiencia multicultural. En N. Collette y A. Hernández (Eds.), Arte, terapia y educación (pp. 79-90). Comunicación presentada en $1^{\text {a }}$ Jornadas de Arte, terapia y educación: La creación como proceso de transformación individual y colectiva. Valencia: Martín Impresores, SL. Universidad Politécnica de Valencia.

Orquillés, P. (2011). Arteterapia en las drogodependencias. Arteterapia: papeles de arteterapia y educación artística para la inclusión social, 6, 135-149.

Öster, I., Svensk, A.C., Magnusson, E., Thyme, K.E., Sjodin, M., Aström, S., y Lindh, J. (2006). Art therapy improves coping resources: a randomized, controlled study among women with breast cancer. Palliate \& Supportive Care, 1, 57-64.

Patterson, S., Debate, J., Anju,S., Waller, D., Crawford, M.J. (2011) Provision and practice of art therapy for people with schizophrenia: results of a national survey. Journal Mental Health, 20(4):328-35. DOI: 10.3109/09638237.2011.556163.

Perry, C., Thurston, M., Osborn, T. (2008). Time for me: the arts as therapy in postnatal depression. Complementary therapies in clinical practice,14(1):38-45. DOI: 10.1016/j.ctcp.2007.06.001. 
Pons-Salvador, G., Frías, D., y Del Barrio, M.V. (1994). Propiedades Psicométricas del State-Trait Anxiety Inventory for Chidren (STAIC). Psicológica, 15, 45-53.

Pratt, R.R. (2004). Art, dance, and music therapy. Physical Medicine and Rehabilitation Clinics of North America, 15, 827-841.

Rico Caballo, L. (2007). Dolor, niños y arte. Arteterapia: papeles de arteterapia y educación artística para la inclusión social, 2, 87-108.

Rodríguez, E. (2007). Aplicaciones del Arteterapia en aula como medio de prevención para el desarrollo de la autoestima y el fomento de las relaciones sociales positivas: "Me siento vivo y convivo "Arteterapia - Papeles de arteterapia y educación artística para la inclusión social, 2, 275-291.

Rousseau C., et al. (2005). Evaluation of a classroom program of creative expression workshops for refugee and immigrant children. Journal of Child Psychology Psychiatry, 46(2): 180-185.

Sánchez, I. (2006). La colección Prinzhorn: una relación falaz entre el arte y la locura. Arte, Individuo y Sociedad, 18, 131-150.

Seisdedos, N. (1990). STAIC, Cuestionario de autoevaluación. Madrid. TEA Ediciones.

Spielberger, C.D., Gorsuch, R., y Lushene, R. (1970). Manual for the State-Trait Anxiety Inventory.Palo Alto, California: Consulting Psychologist Press.

Svensk, A.C., Öster, I., Thyme, K.E., Magnusson, E., Sjodin, M., Eisemann, M., Aström, S., y Lindh, J. (2009). Art therapy improves experienced quality of live among women undergoing treatment for breast cancer: a randomized controlled study. European Journal Cancer Care, 18, 69-77.

Thurstone,L.L., Yela, M. (2001). CARAS: Test de percepción de diferencias. Madrid: TEA Ediciones.

Walsh, S.M., Radcliffe, R.S., Castillo, L.C., Kumar, A.M., y Broschard D.M. (2007). A pilot study to test the effects of art-making classes for family caregivers of patients with cancer. Oncology Nursing Forum, 34, 38.

Wilcutt, EG., et al. (2005). Validity of executive function theory of attention- deficit/ hyperactivity disorder: A meta-analytic review. Biological Psychiatry,57,1336-1346.

Wood, M., Molassiotis, A., y Payne S. (2011). What research evidence is there for the use of arttherapy in the management of symptoms in adults with cancer? A systematic review. Psychooncology, 20, 135-145.

Zubillaga, A. y Pastor, C. (2005). Las Webquest como recurso educativo para alumnos con necesidades educativas especiales. Comunicación y Pedagogía, 206, 50-57. 